\title{
Numerical Solution of Linear and Nonlinear Integral Equations Via Improved Block-Pulse Functions
}

\author{
Mahmoud Hamed Taha ${ }^{1}$, Mohamed Abdel-Latif Ramadan², Galal Mahrous Moatimid \\ ${ }^{1}$ Department of Mathematics, Faculty of Education, Ain Shams University, Roxy, Cairo, Egypt \\ ${ }^{2}$ Department of Mathematics and Computer Science, Faculty of Science, Menoufia University, Menoufia, Egypt
}

Email address:

mahmoud.h.taha@edu.asu.edu.eg (M.H.Taha),ramadanmohamed13@yahoo.com (M. Abdel-Latif R.), mramadan@eun.eg (M. Abdel-Latif R.),Gal_moa@edu.asu.edu.eg (G. M. Moatimid)

\section{To cite this article:}

Mahmoud Hamed Taha, Mohamed Abdel-Latif Ramadan, Galal Mahrous Moatimid. Numerical Solution of Linear and Nonlinear Integral Equations Via Improved Block-Pulse Functions. American Journal of Mathematical and Computer Modelling. Vol. 6, No. 2, 2021, pp. 19-34. doi: 10.11648/j.ajmcm.20210602.11

Received: March 6, 2021; Accepted: March 22, 2021; Published: May 26, 2021

\begin{abstract}
This paper is concerned with a numerical method based on the improved block-pulse basis functions (IBPFs). It is done mainly to solve linear and nonlinear Volterra and Fredholm integral equations of the second kind. These equations can be simplified into a linear system of algebraic equations by using IBPFs and their operational matrix of integration. After that, the system can be programmed and solved using Mathematica. The changes made to the method obviously improved - as it will be shown in the numerical examples - the time taken by the program to solve the system of algebraic equations. Also, it is reflected in the accuracy of the solution. This modification works perfectly and improved the accuracy over the regular block-pulse basis functions (BPF). A slight change in the intervals of the BPF changes the whole technique to a new easier and more accurate technique. This change has worked well while solving different types of integral equations. The accompanied theorems of the IBPF technique and error estimation are stated and proved. The paper also dealt with the uniqueness and convergence theorems of the solution. Numerical examples are presented to illustrate the efficiency and accuracy of the method. The tables and required graphs are also shown to prove and demonstrate the efficiency.
\end{abstract}

Keywords: Linear Integral Equations, Nonlinear Integral Equations, Improved Block-Pulse Functions, Operational Matrix, Vector Forms, Error Analysis

\section{Introduction}

In recent years, there has been a growing interest in the formulation of many engineering and physical problems in terms of integral equations. This has been fostered a parallel rapid growth of the literature on their numerical solution. Therefore, this article gives a further contribution to what is becoming a subject of increasing concern to scientists, engineers, and mathematicians. Moreover, linear/nonlinear integral equations are typically hard to solve analytically, however; some numerical techniques are used to solve these equations.

During the last decade, many attempts have been made to solve linear/nonlinear integral equations by several researchers by using numerical or perturbed methods. The time collocation method and the projection method did a great job in solving it [1]. Others applied an iterative method to solve this equation such as the Lagrange method [2], modified Homotopy perturbation method [3], rationalized Haar function method [4, 5], differential transformation method [6], and Tau method [7]. In 1999, He [8] tried to solve linear differential and integral equations by using a new method which is called (HPM). Furthermore, he managed to solve some nonlinear problems [9]. After that, he developed his technique to work on more complicated problems and introduced a solution to a lot of applications [10]. The Adomian decomposition method is used by Wazwaz [11] with a lot of modifications to the method. He managed to solve and get an approximate solution for most cases of that equation.

In this paper, IBPFs are presented. Moreover, some theorems are proved for the IBPFs method which shows some results of these numerical expansions, that are more precise than the results of the block pulse expansions. These functions are disjoint, orthogonal, and complete. According to the 
disjointness of IBPFs, the joint terms will be disappeared in each subinterval when multiplication, division, and some other operations are applied. Additionally, the orthogonality property of IBPFs will cause an operational matrix to be sparse. The completeness of IBPFs guarantees an arbitrary small mean square error that can be obtained from a real bounded function. This has only a finite number of discontinuous points in the interval $\mathrm{x} \in[0,1)$ by increasing the number of terms in the improved block pulse series. To crystallize the presentation of the current paper, the rest of it is organized as follows: In Section 2, we describe IBPFs and their properties, also how to apply them to linear and nonlinear different types of integral equations. Convergence analysis is discussed in Section 3. Numerical results are given in Section 4 to illustrate the efficiency and accuracy of the proposed method. Finally, Section 5gives the main outlines of the paper as a conclusion.

\section{The Basic Idea of the New Improved Block Pulse Function (IBPF)}

Suppose the integral equations in the forms of the following Fredholm integral equation:

$$
y(t)=f(t)+\int_{0}^{1} k_{1}(t, s) .(y(s))^{r} d s, t \in[0,1),
$$

or in a nonlinear version

$$
y(t)=f(t)+\int_{0}^{x} k_{2}(t, s) \cdot(y(s))^{q} d s, t \in[0,1) .
$$

where $y(t)$ is the function to be determined, $f(t)$ is an analytical function over the interval $[0,1), k_{1}(t, s)$ and $k_{2}(t, s)$ are called the kernels of the integral equation. They are analytic on $[0,1) \times[0,1), \mathrm{r}$, and $\mathrm{q}$ are non-negative integers. All these functions may be represented in a vector form respectively as follows:

$$
\begin{aligned}
Y_{m} & =\left[y_{0}(t), y_{1}(t), \ldots, y_{m}(t)\right]^{T}, \\
F_{m} & =\left[f_{0}(t), f_{1}(t), \ldots, f_{m}(t)\right]^{T},
\end{aligned}
$$

and

$$
K_{m}=\left[k_{i j}(t, s)\right], i, j=0,1, \ldots, m .
$$

The IBPFs were firstly introduced by Farshid Mirzaee [12]. Farshid solved a system of integral equations by using IBPFs. However; in this article, an operator matrix of the Volterra integral equation is modified to get better results. Here linear and nonlinear integral equations will be solved. In addition, mixed Fredholm and Volterra integral equations will be considered. The variable interval block pulse functions are derived from the regular block pulse functions, but with a slight change in interval width. This change caused a huge alteration in the algorithm of the method which we will study in detail. The variable of interval block pulse is a $(m+1)$ agreed of functions defined over the interval $[0,1)$ as follows:

$$
\begin{gathered}
\varphi_{0}(t)=\left\{\begin{array}{l}
1, t \in\left[0, \frac{1}{2 m}\right), \\
0, \text { otherwise },
\end{array}\right. \\
\varphi_{i}(t)=\left\{\begin{array}{l}
1, t \in\left[\frac{i-\frac{1}{2}}{m}, \frac{i+\frac{1}{2}}{m}\right), i=1,2, \ldots, m-1 \\
0, \text { otherwise }
\end{array}\right. \\
\text { and } \varphi_{m}(t)=\left\{\begin{array}{l}
1, t \in\left[1-\frac{1}{2 m}, 1\right), \\
0, \text { otherwise },
\end{array}\right.
\end{gathered}
$$

where $m$ is a positive constant represents the number of sub-interval to be decided for the accuracy needed in solving the problem. The IBPFs are disjointas

$$
\varphi_{i}(t) \varphi_{j}(t)=\left\{\begin{array}{l}
\varphi_{i}(t), i=j \\
0, \text { otherwise }
\end{array}\right.
$$

where $i, j=0,1, \ldots, m$ and orthogonal to each other

$$
\int_{0}^{1} \varphi_{i}(t) \varphi_{j}(t) d t=\left\{\begin{array}{l}
\frac{1}{2 m}, i=j \in\{0, m\} \\
\frac{1}{m}, i=j \in\{1,2, \ldots, m-1\} \\
0, \text { otherwise }
\end{array}\right.
$$

where $t \in[0,1)$.

The first $(m+1)$ terms of the IBPF can be written in vector form

$$
\phi_{m}(t)=\left[\varphi_{0}(t), \varphi_{1}(t), \ldots, \varphi_{m}(t)\right]^{T}, t \in[0,1)
$$

Eq. (9) gives

$$
\phi_{m}(t) \phi_{m}^{T}(t)=\left[\begin{array}{llll}
\varphi_{0}(t) & 0 & \cdots & 0 \\
0 & \varphi_{1}(t) & \cdots & 0 \\
\vdots & \vdots & \ddots & \vdots \\
0 & 0 & \cdots & \varphi_{m}(t)
\end{array}\right]=
$$

Furthermore, one gets

$$
\begin{gathered}
\int_{0}^{t} \varphi_{0}(s) d s=\left\{\begin{array}{l}
t, t \in\left[0, \frac{1}{2 m}\right), \\
\frac{1}{2 m}, \text { otherwise, }
\end{array}\right. \\
\int_{0}^{t} \varphi_{i}(s) d s=\left\{\begin{array}{l}
0, t \in\left[0, \frac{i-1 / 2}{m}\right), \\
t-\left(\frac{i-\frac{1}{2}}{m}\right), t \in\left[\frac{i-1 / 2}{m}, \frac{i+1 / 2}{m}\right), \\
\frac{1}{m}, \text { otherwise },
\end{array}\right. \\
\int_{0}^{t} \varphi_{n}(s) d s=\left\{\begin{array}{l}
t-\left(1-\frac{1}{2 m}\right), t \in\left[1-\frac{1}{2 m}, 1\right), \\
0, \text { otherwise. }
\end{array}\right.
\end{gathered}
$$

It is worthy to note that at the first and last interval $t=\frac{1}{4 m}$. So, it can be approximated by this value where $m=0$ or $n$. However, at any of the middle interval $t-\left(\frac{i-1 / 2}{m}\right)$ can be approximated by $\frac{1}{2 m}$. Then we can deduce that the integration of the vector $\phi_{m}(t)$ defined in Eq. (11) can be represented approximately as 


$$
\int_{0}^{t} \phi_{m}(s) d s \simeq B \phi_{m}(t)
$$

where

$$
B=\frac{1}{m}\left[\begin{array}{cccccc}
\frac{1}{2} & 1 & 1 & \cdots & 1 & 1 \\
0 & \frac{1}{2} & 1 & \cdots & 1 & 1 \\
0 & 0 & \frac{1}{2} & \cdots & 1 & 1 \\
\vdots & \vdots & \vdots & \ddots & \vdots & \vdots \\
0 & 0 & 0 & \cdots & \frac{1}{2} & 1 \\
0 & 0 & 0 & \cdots & 0 & \frac{1}{2}
\end{array}\right] .
$$

This operational matrix may be modified and rewritten to get better results than that used in the work of F. Mirzaee, [12]. By using the relation given in Eq. (12), one gets

$$
\int_{0}^{1} \phi_{m}(s) \phi_{m}^{T}(s) d s \simeq V
$$

where

$$
V=\frac{1}{2 m}\left[\begin{array}{llllll}
1 & 0 & 0 & \cdots & 0 & 0 \\
0 & 2 & 0 & \cdots & 0 & 0 \\
0 & 0 & 2 & \cdots & 0 & 0 \\
\vdots & \vdots & \vdots & \ddots & \vdots & \vdots \\
0 & 0 & 0 & \cdots & 2 & 0 \\
0 & 0 & 0 & \cdots & 0 & 1
\end{array}\right]
$$

Suppose $y(t)$ is a continuous function, where $y(t) \in$ $L^{2}([0,1))$ and maybe expanded by the IBPFs as:

$$
y(t) \simeq y_{m}(t)=\sum_{i=0}^{m} y_{i} \varphi_{i}(t)=Y_{m}^{T} \phi_{m}(t)=\phi_{m}^{T}(t) Y_{m} .
$$

Only m-terms of Eq. (20) are considered, where $\mathrm{m}$ is a power of 2 , and $Y_{m}$ is an $(m+1) \times 1$ vector given by

$$
Y_{m}=\left[y_{0}, y_{1}, \cdots, y_{m}\right]^{T},
$$

In addition, $f(t)$ can be expanded by the IBPFs as

$$
f(t) \simeq f_{m}(t)=\sum_{i=0}^{m} f_{i} \varphi_{i}(t)=F_{m}^{T} \phi_{m}(t)=\phi_{m}^{T}(t) F_{m},
$$

where $F_{m}$ is an $(m+1) \times 1$ vector given by

$$
F_{m}=\left[f_{0}, f_{1}, \cdots, f_{m}\right]^{T},
$$

and $\phi_{m}(t)$ is defined in Eq. (11), and $y_{i}$ are the improved block pulse coefficients and are obtained by

$$
y_{i}=\left\{\begin{array}{l}
2 m \int_{0}^{1 / 2 m} y(t) d t, i=0, \\
m \int_{\frac{(i+1 / 2) / m}{m}}^{\frac{(i-1 / 2) / m}{m}} y(t) d t, 1 \leq i \leq m-1 . \\
2 m \int_{1-1 / 2 m}^{1} y(t) d t, i=m .
\end{array}\right.
$$

Similarly a function of two variables, $k(t, s) \in L^{2}([0,1) \times$ $[0,1))$ can be approximated by IBPFs as follows

$$
k(t, s) \simeq k_{m}(t, s)=\phi_{m}^{T}(t) K_{m} \phi_{m}(s) .
$$

\subsection{Solution Algorithm for Fredholm Integral Equation}

Combining Eq. (20), Eq. (22) and Eq. (25) into Eq. (1) with $\mathrm{r}=1$, one finds

$$
\phi_{m}^{T}(t) Y_{m}=\phi_{m}^{T}(t) F_{m}+\int_{0}^{1} \phi_{m}^{T}(t) K_{m} \phi_{m}(s) \phi_{m}^{T}(s) Y_{m} d s .
$$

From Eq. (12), one gets

$\phi_{m}^{T}(t) Y_{m}=\phi_{m}^{T}(t) F_{m}+\int_{0}^{1} \phi_{m}^{T}(t) K_{m} \operatorname{diag}\left(\phi_{m}(s)\right) Y_{m} d s$

or

$$
\begin{gathered}
\phi_{m}^{T}(t) Y_{m}=\phi_{m}^{T}(t) F_{m}+\phi_{m}^{T}(t) K_{m} \int_{0}^{1} \operatorname{diag}\left(\phi_{m}(s)\right) d s Y_{m} \\
\phi_{m}^{T}(t) Y_{m}=\phi_{m}^{T}(t) F_{m}+\phi_{m}^{T}(t) K_{m} V Y_{m}
\end{gathered}
$$

From Eq. (18), one obtains

$$
\int_{0}^{1} \operatorname{diag}\left(\phi_{m}(s)\right) d s \simeq V .
$$

It follows that

$$
Y_{m}=F_{m}+K_{m} V Y_{m}
$$

Therefore, the unknown coefficients $Y_{i}$ can be determined from the relation

$$
Y_{m}=\left[I_{m+1}-A\right]^{-1} F_{m}
$$

Hence, $I_{m+1}$ is the identity matrix with dimensions $(m+1) \times(m+1)$, and $A=K_{m} V$. It can be calculated by using Eq. (19) as follows:

$$
\begin{aligned}
A & =\left[\begin{array}{lllll}
k_{00} & k_{01} & k_{02} & \cdots & k_{0 m} \\
k_{10} & k_{11} & k_{12} & \cdots & k_{1 m} \\
k_{20} & k_{21} & k_{22} & \cdots & k_{2 m} \\
\vdots & \vdots & \vdots & \ddots & \vdots \\
k_{m 0} & k_{m 1} & k_{m 2} & \cdots & k_{m m}
\end{array}\right] \cdot \frac{1}{2 m}\left[\begin{array}{llllll}
1 & 0 & 0 & \cdots & 0 & 0 \\
0 & 2 & 0 & \cdots & 0 & 0 \\
0 & 0 & 2 & \cdots & 0 & 0 \\
\vdots & \vdots & \vdots & \ddots & \vdots & \vdots \\
0 & 0 & 0 & \cdots & 2 & 0 \\
0 & 0 & 0 & \cdots & 0 & 1
\end{array}\right], \\
& =\frac{1}{m}\left[\begin{array}{llllll}
\frac{1}{2} k_{00} & k_{01} & k_{02} & \cdots & \frac{1}{2} k_{0 m} \\
\frac{1}{2} k_{10} & k_{11} & k_{12} & \cdots & \frac{1}{2} k_{1 m} \\
\frac{1}{2} k_{20} & k_{21} & k_{22} & \cdots & \frac{1}{2} k_{2 m} \\
\vdots & \vdots & \vdots & \ddots & \vdots \\
\frac{1}{2} k_{m 0} & k_{m 1} & k_{m 2} & \cdots & \frac{1}{2} k_{m m}
\end{array}\right]_{m+1 \times m+1}
\end{aligned}
$$

where $k i j$ can be computed in the following manner

$$
\begin{aligned}
k_{00} & =4 m^{2} \int_{0}^{1 / 2 m} \int_{0}^{1 / 2 m} k(x, t) d x d t ; \\
k_{m 0} & =4 m^{2} \int_{0}^{1 / 2 m} \int_{1-1 / 2 m}^{1} k(x, t) d x d t \\
k_{0 m} & =4 m^{2} \int_{1-1 / 2 m}^{1} \int_{0}^{1 / 2 m} k(x, t) d x d t ;
\end{aligned}
$$




$$
\begin{gathered}
k_{i j}=m^{2} \int_{\frac{j-1 / 2}{m} \frac{j-1 / 2}{m}}^{\frac{j+1 / 2}{m}} k(x, t) d x d t \\
k_{i m}=2 m^{2} \int_{\frac{i-1 / 2}{m}}^{\frac{i+1 / 2}{m}} \int_{1-1 / 2 \mathrm{~m}}^{1} k(x, t) d x d t ; \\
k_{m j}=2 m^{2} \int_{1-1 / 2 \mathrm{~m}}^{1} \frac{\int_{i-1 / 2}^{m}}{m} k(x, t) d x d t \\
k_{i 0}=2 m^{2} \int_{\frac{j-1 / 2}{m}}^{\frac{j+1 / 2}{m}} \int_{0}^{\frac{1}{m}} k(x, t) d x d t ; \\
k_{0 j}=2 m^{2} \int_{0}^{\frac{1}{2 m}} \frac{i+1 / 2}{m} k(x, t) d x d t \\
\int_{-1 / 2}^{m}
\end{gathered}
$$

By solving the linear system in Eq. (32), $Y_{m}$ may be obtained to get

$$
y(t) \simeq \sum_{i=0}^{m} y_{i} \varphi_{i}(t)
$$

\subsection{Solution Algorithm for Volterra Integral Equation}

Combining Eq. (20), Eq. (22) and Eq. (25) into Eq. (2) with $\mathrm{q}=1$, one gets

$\phi_{m}^{T}(t) Y_{m}=\phi_{m}^{T}(t) F_{m}+\int_{0}^{t} \phi_{m}^{T}(t) K_{m} \phi_{m}(s) \phi_{m}^{T}(s) Y_{m} d s$
$\phi_{m}^{T}(t) Y_{m}=\phi_{m}^{T}(t) F_{m}+\int_{0}^{t} \phi_{m}^{T}(t) K_{m} \operatorname{diag}\left(\phi_{m}(s)\right) Y_{m} d s$

or

$$
\begin{gathered}
\phi_{m}^{T}(t) Y_{m}=\phi_{m}^{T}(t) F_{m}+\phi_{m}^{T}(t) K_{m} \int_{0}^{t} \operatorname{diag}\left(\phi_{m}(s)\right) d s Y_{m} \\
\phi_{m}^{T}(t) Y_{m}=\phi_{m}^{T}(t) F_{m}+\phi_{m}^{T}(t) K_{m} B Y_{m}
\end{gathered}
$$

Assuming that

$$
B=\left[\begin{array}{lllll}
H_{0} \phi_{m}(t) & 0 & 0 & \cdots & 0 \\
0 & H_{1} \phi_{m}(t) & 0 & \cdots & 0 \\
0 & 0 & H_{2} \phi_{m}(t) & \cdots & 0 \\
\vdots & \vdots & \vdots & \ddots & \vdots \\
0 & 0 & 0 & \cdots & H_{m} \phi_{m}(t)
\end{array}\right]
$$

where $H_{i}$ is the ith row of $H$, it follows that

$$
Y_{m}=F_{m}+K_{m} B Y_{m}
$$

Therefore, the unknown coefficients $Y_{i}$ may be determined from the following relation:

$$
Y_{m}=\left[I_{m+1}-K_{m} B\right]^{-1} F_{m},
$$

where $I_{m+1}$ is the identity matrix with dimensions $(m+$ $1) \times(m+1)$, and $K_{m} B$ can be calculated by using Eq. (17) as follows:

From Eq. (10), one finds

$$
K_{m} B=\left[\begin{array}{lllll}
k_{00} & k_{01} & k_{02} & \cdots & k_{0 m} \\
k_{10} & k_{11} & k_{12} & \cdots & k_{1 m} \\
k_{20} & k_{21} & k_{22} & \cdots & k_{2 m} \\
\vdots & \vdots & \vdots & \ddots & \vdots \\
k_{m 0} & k_{m 1} & k_{m 2} & \cdots & k_{m m}
\end{array}\right]\left[\begin{array}{lllll}
H_{0} \phi_{m}(t) & 0 & 0 & \cdots & 0 \\
0 & H_{1} \phi_{m}(t) & 0 & \cdots & 0 \\
0 & 0 & H_{2} \phi_{m}(t) & \cdots & 0 \\
\vdots & \vdots & \vdots & \ddots & \vdots \\
0 & 0 & 0 & \cdots & H_{m} \phi_{m}(t)
\end{array}\right],
$$

In the same manner, $[y(t)]^{r}$ can be approximated in terms

$$
=\frac{1}{m}\left[\begin{array}{lllll}
\frac{1}{2} k_{00} & k_{01} & k_{02} & \cdots & k_{0 m} \\
0 & \frac{1}{2} k_{11} & k_{12} & \cdots & k_{1 m} \\
0 & 0 & \frac{1}{2} k_{22} & \cdots & k_{2 m} \\
\vdots & \vdots & \vdots & \ddots & \vdots \\
0 & 0 & 0 & \cdots & \frac{1}{2} k_{m m}
\end{array}\right]_{m+1 \times m+1} .
$$

Solving the linear system in Eq. (44) $Y_{m}$ may be obtained to get

$$
y(t) \simeq \sum_{i=0}^{m} y_{i} \varphi_{i}(t) .
$$

\subsection{Solution Algorithm for Non-linear Integral Equations}

In this section, the non-linear Volterra-Fredholm integral equation given in Eq. (2) will be solved by using BPF. As mentioned in the previous sections, it is seen that $y(t)$ is a function defined over the interval $[0,1)$ maybe expanded as

$$
[y(t)]^{r} \simeq \tilde{Y}_{m}^{T} \phi_{m}(t) .
$$

Now, the vector $\tilde{Y}$ needs to be calculated. So, one gets

$$
y(t)=Y_{m}^{T} \phi_{m}(t) \text { and }[y(t)]^{r} \simeq \tilde{Y}_{m}^{T} \phi_{m}(t)
$$

Therefore, one gets

$$
\tilde{Y}_{m}^{T} \phi(t)=\left[Y_{m}^{T} \phi_{m}(t)\right]^{r}
$$

Hence, from Eq. (9), Eq. (12) and Eq. (19)

$$
\int_{0}^{1} \varphi_{i}(t) \varphi_{j}(t) d t=\left\{\begin{array}{l}
\frac{1}{2 m}, i=j \in 0, m, \\
\frac{1}{m}, i=j \in 1,2, \ldots, m-1, \\
0, \text { otherwise, }
\end{array}\right.
$$

$$
y(t) \simeq y_{m}(t)=\sum_{i=0}^{m} y_{i} \varphi_{i}(t)=Y_{m}^{T} \phi_{m}(t)=\phi_{m}^{T}(t) Y_{m}
$$




$$
\phi_{m}(t) \phi_{m}^{T}(t)=\left[\begin{array}{llll}
\varphi_{0}(t) & 0 & \cdots & 0 \\
0 & \varphi_{1}(t) & \cdots & 0 \\
\vdots & \vdots & \ddots & \vdots \\
0 & 0 & \cdots & \varphi_{m}(t)
\end{array}\right]=
$$

So, one finds

$$
\begin{gathered}
\int_{0}^{1} \phi_{m}(t) \phi_{m}^{T}(t) d t=\frac{1}{m}\left[\begin{array}{cccccc}
\frac{1}{2} & 0 & 0 & \cdots & 0 & 0 \\
0 & 1 & 0 & \cdots & 0 & 0 \\
0 & 0 & 1 & \cdots & 0 & 0 \\
\vdots & \vdots & \vdots & \ddots & \vdots & \vdots \\
0 & 0 & 0 & \cdots & 1 & 0 \\
0 & 0 & 0 & \cdots & 0 & \frac{1}{2}
\end{array}\right]=\frac{1}{m} P_{m+1} \\
\int_{0}^{1} \phi_{m}(t) \phi_{m}^{T}(t) d t=\frac{1}{m} P_{m+1} \\
m P_{m+1}^{-1} \int_{0}^{1} \phi_{m}(t) \phi_{m}^{T}(t) d t=I_{m+1}
\end{gathered}
$$

where $P_{m+1}^{-1}$ is the inverse of the matrix $P_{m+1}$, and $I_{m+1}$ is

$$
\begin{aligned}
& \tilde{Y}_{m}^{T}=m P_{m+1}^{-1}\left[\begin{array}{l}
\int_{0}^{1 / 2 m}\left[Y_{m}^{T} \phi_{m}(t)\right]^{r-1} Y_{m}^{T}\left[\phi_{m}(t) \phi_{m}^{T}(t)\right] d t \\
+\sum_{i=1}^{m-1} \int_{\frac{i-1 / 2}{m}}^{\frac{i+1 / 2}{m}}\left[Y_{m}^{T} \phi_{m}(t)\right]^{r-1} Y_{m}^{T}\left[\phi_{m}(t) \phi_{m}^{T}(t)\right] d t \\
+\int_{1-\frac{1}{2 m}}^{1}\left[Y_{m}^{T} \phi_{m}(t)\right]^{r-1} Y_{m}^{T}\left[\phi_{m}(t) \phi_{m}^{T}(t)\right] d t
\end{array}\right] \\
& \tilde{Y}_{m}^{T}=m P_{m+1}^{-1}\left[\int_{0}^{1 / 2 m}\left[\left[y_{0}, y_{1}, \cdots, y_{m}\right]\left(\begin{array}{c}
1 \\
0 \\
: \\
0
\end{array}\right)\right]^{r-1} \cdot\left[y_{0}, y_{1}, \cdots, y_{m}\right]\left(\begin{array}{cccc}
1 & 0 & . . & 0 \\
0 & 0 & 0 & 0 \\
: & 0 & \ddots & : \\
0 & 0 & . . & 0
\end{array}\right) d t\right] \\
& + \\
& \left.+m P_{m+1}^{-1}\left[\int_{\frac{i-1 / 2}{m}}^{\frac{i+1 / 2}{m}}\left[y_{0}, y_{1}, \cdots, y_{m}\right]\left(\begin{array}{c}
0 \\
1 \\
\vdots \\
0
\end{array}\right)\right]^{r-1} \cdot\left[y_{0}, y_{1}, \cdots, y_{m}\right]\left(\begin{array}{cccc}
0 & 0 & . . & 0 \\
0 & 1 & 0 & 0 \\
: & 0 & \ddots & : \\
0 & 0 & . . & 0
\end{array}\right) d t\right] \\
& +m P_{m+1}^{-1}\left[\int_{1-1 / 2 m}^{1}\left[\left[y_{0}, y_{1}, \cdots, y_{m}\right]\left(\begin{array}{c}
0 \\
0 \\
\vdots \\
1
\end{array}\right)\right]^{r-1} \cdot\left[y_{0}, y_{1}, \cdots, y_{m}\right]\left(\begin{array}{cccc}
0 & 0 & . . & 0 \\
0 & 0 & 0 & 0 \\
: & 0 & \ddots & : \\
0 & 0 & . . & 1
\end{array}\right) d t\right] \\
& =m P_{m+1}^{-1}\left[\int_{0}^{1 / 2 m}\left[y_{0}\right]^{r-1} \cdot\left[y_{0}, 0, \cdots, 0\right] d t+\cdots+\int_{\frac{i-1 / 2}{m}}^{\frac{i+1 / 2}{m}}\left[y_{i}\right]^{r-1} \cdot\left[0, y_{i}, \cdots, 0\right] d t+\cdots+\int_{1-1 / 2 m}^{1}\left[y_{m}\right]^{r-1} \cdot\left[0,0, \cdots, y_{m}\right] d t\right]
\end{aligned}
$$




$$
\begin{gathered}
=m P_{m+1}^{-1}\left[\int_{0}^{1 / 2 m}\left[y_{0}^{r}, 0, \cdots, 0\right] d t+\cdots+\int_{\frac{i-1 / 2}{m}}^{\frac{i+1 / 2}{m}}\left[0, y_{i}^{r}, \cdots, 0\right] d t+\cdots+\int_{0}^{1 / m}\left[0,0, \cdots, y_{m}^{r}\right] d t\right] \\
=m P_{m+1}^{-1}\left[\frac{1}{2 m}\left[y_{0}^{r}, 0, \cdots, 0\right]+\cdots+\frac{1}{m}\left[0, y_{i}^{r}, \cdots, 0\right]+\cdots+\frac{1}{2 m}\left[0,0, \cdots, y_{m}^{r}\right]\right]=P_{m+1}^{-1}\left[2 y_{0}^{r}, y_{1}^{r}, \cdots, 2 y_{m}^{r}\right]=\left[y_{0}^{r}, y_{1}^{r}, \cdots, y_{m-1}^{r}, y_{m}^{r}\right] .
\end{gathered}
$$

Now, in order to solve the nonlinear Volterra-Fredholm integral equation given in Eq. (2), the following approximations must be used

$$
\begin{gathered}
y(t) \simeq \phi_{m}^{T}(t) Y_{m}, \\
f(t) \simeq \phi_{m}^{T}(t) F_{m}, \\
y(t)]^{r} \simeq \tilde{Y}_{1}^{T} \phi_{m}(t), \\
y(t)]^{q} \simeq \tilde{Y}_{2}^{T} \phi_{m}(t), \\
k_{1}(t, s) \simeq \phi_{m}^{T}(t) K_{1} \phi_{m}(s), \\
k_{2}(t, s) \simeq \phi_{m}^{T}(t) K_{2} \phi_{m}(s),
\end{gathered}
$$

where the $m+1$ vectors $Y_{m}, F_{m}, \tilde{Y}_{1}^{T}, \tilde{Y}_{2}^{T}$ and the $(m+1) \times$ $(m+1)$ matrices $K_{1}$ and $K_{2}$ are the IBPFs coefficients the non-linear equation

$$
\begin{aligned}
y(t) & =f(t)+\int_{0}^{1} k_{1}(t, s) \cdot(y(s))^{r} d s \\
& +\int_{0}^{x} k_{2}(t, s) \cdot(y(s))^{q} d s, t \in[0,1) .
\end{aligned}
$$

It is transferring to

$$
\begin{gathered}
\phi_{m}^{T}(t) Y_{m}=\phi_{m}^{T}(t) F_{m}+\int_{0}^{1} \phi_{m}^{T}(t) K_{1} \phi_{m}(s) . \tilde{Y}_{1}^{T} \phi_{m}(s) d s \\
+\int_{0}^{x} \phi_{m}^{T}(t) K_{2} \phi_{m}(s) . \tilde{Y}_{2}^{T} \phi_{m}(s) d s, t \in[0,1),
\end{gathered}
$$

or

$$
\begin{gathered}
\phi_{m}^{T}(t) Y_{m}=\phi_{m}^{T}(t) F_{m}+\phi_{m}^{T}(t) K_{1} \int_{0}^{1} \phi_{m}(s) \cdot \phi_{m}^{T}(s) d s \tilde{Y}_{1} \\
+\phi_{m}^{T}(t) K_{2} \int_{0}^{x} \phi_{m}(s) \cdot \phi_{m}^{T}(s) d s \tilde{Y}_{2}, t \in[0,1),
\end{gathered}
$$

which will give the following the linear system

$$
Y_{m}=F_{m}+K_{1} V \tilde{Y}_{1}+K_{2} B \tilde{Y}_{2},
$$

Solving this system in Eq. (72), $Y_{m}$ can be found and then we can find $y_{i}$ and to get the solution substitute in

$$
y(t) \simeq \sum_{i=0}^{m} y_{i} \varphi_{i}(t) .
$$

\section{Convergence Analysis}

In this section, we show that the current method is convergent. Its order of convergence is $O\left(\frac{1}{m}\right)$ or $O(h)$. We define

$$
\begin{aligned}
& \|y(x)\|=\left(\int_{0}^{1}|y(x)|^{2} d x\right)^{1 / 2}, \\
& \text { and }\|Y(x)\|=\left(\sum_{i=1}^{m}\left\|Y_{i}(x)\right\|^{2}\right)^{1 / 2},
\end{aligned}
$$

where $y(x) \in L^{2}(D)$ and $Y(x)$ is defined as in Eq. (2), and

$$
\begin{aligned}
\|k(x, y)\| & =\left(\int_{0}^{1} \int_{0}^{1}|k(x, y)|^{2} d x d y\right)^{1 / 2}, \\
\text { and }\|k(x, y)\| & =\left(\sum_{i=0}^{m} \sum_{j=0}^{m}\left\|k_{i j}(x, y)\right\|^{2}\right)^{1 / 2},
\end{aligned}
$$

where $k(x, y) \in L^{2}(D \times D)$ and $k(x, y)$ is defined in Eq. (7).

For this purpose, we will need to prove the following theorems:

Theorem 1

Let $y(x) \in L^{2}(D)$ and $y_{m}(x)$ be the IBPFs expansion of $y(x)$ that is defined as

$$
y_{m}(x)=\sum_{i=0}^{m} y_{i} \varphi_{i}(t)
$$

where $y_{i} ; \mathrm{i}=0,1, \ldots, \mathrm{m}$, are defined as in Eq. (24). Therefore, the criterion of this approximation mean square error between the functions $y(x)$ and $y_{m}(x)$ in the interval $x \in D$ as

$$
\int_{0}^{1}\left(y(x)-y_{m}(x)\right)^{2} d x
$$

achieves its minimum value and also

$$
\int_{0}^{1}(y(x))^{2} d x=\sum_{i=0}^{\infty} y_{i}^{2}\left\|\phi_{i}(x)\right\|^{2}
$$

Proof

It is an immediate consequence of the theorem which is proved in the work of Jiang and Schaufelberger, [13].

Theorem 2

Supposethat $y(x)$ is a continuous on $D$, differentiable on $(0,1)$, and there exists a positive scalar M such that $\left|y^{\prime}(x)\right| \leq$ $M$, for every $x \in D$. Then

$$
|y(b)-y(a)| \leq M|b-a|, \forall a, b \in D,
$$

Proof see Ref.[14].

Theorem 3 
Suppose that $y_{m}(x)$ is the IBPFs expansion of $y(x)$, defined as Eq. (22) and $f(x)$ is differentiable on $D$ such that $\left|y^{\prime}(x)\right| \leq M$.

Also, assume that $e_{m}(x)=y(x)-y_{n}(x)$, therefore, one gets

$$
\left\|e_{m}(x)\right\|=O(h)
$$

Proof

Suppose $x_{0}=0, x_{i}=i h-\frac{h}{2}, i=1, \ldots, m$ and $x_{m+1}=1$. We define the error between the function $y(x)$ and its IBPFs expansion over every subinterval $I_{i}=\left[x_{i}, x_{i+1}\right)$ as follows:

$$
e_{m, i}(x)=y(x)-y_{i}(x), x \in I_{i}
$$

where $i=0,1, \ldots, m$.

By using the mean value theorem for integrals, one finds

$$
\left\|e_{m, 0}(x)\right\|^{2}=\int_{0}^{\frac{h}{2}} e_{m, 0}^{2}(x) d x=\int_{0}^{\frac{h}{2}}\left(y(x)-y_{0}\right)^{2} d x=
$$

where $\varepsilon_{0} \in I_{0}$. Also, for $i=1,2, \ldots, m-1$, we have

$$
\left\|e_{m, i}(x)\right\|^{2}=\int_{i h-\mathrm{h} / 2}^{i h+\mathrm{h} / 2} e_{m, i}^{2}(x) d x=\int_{i h-\mathrm{h} / 2}^{i h+\mathrm{h} / 2}\left(y(x)-y_{i}\right)^{2} d x=
$$

where $\varepsilon_{i} \in I_{i}$. Furthermore, we have

$$
\begin{gathered}
\left\|e_{m, m}(x)\right\|^{2}=\int_{1-\mathrm{h} / 2}^{1} e_{m, m}^{2}(x) d x=\int_{1-\mathrm{h} / 2}^{1}\left(y(x)-y_{m}\right)^{2} d x= \\
\frac{h}{2}\left(y\left(\varepsilon_{m}\right)-y_{m}\right)^{2},
\end{gathered}
$$

where $\varepsilon_{m} \in I_{m}$.

By using Eq. (24) and the mean value theorem, we have

$$
y_{i}=\left\{\begin{array}{l}
2 m \int_{0}^{1 / 2 m} y(t) d t=2 m \frac{h}{2} y\left(\varepsilon_{0}\right)=y\left(\varepsilon_{0}\right), i=0, \\
m \int_{\frac{i-1 / 2}{m}}^{\frac{i+1 / 2}{m}} y(t) d t=m h y\left(\varepsilon_{i}\right)=y\left(\varepsilon_{i}\right), 1 \leq i \leq m-1, \\
2 m \int_{1-1 / 2 \mathrm{~m}}^{1} y(t) d t=2 m \frac{h}{2} y\left(\varepsilon_{m}\right)=y\left(\varepsilon_{m}\right), i=m .
\end{array}\right.
$$

where $\varepsilon_{i} \in I_{i}, i=0,1, \ldots, m$.

From the above equations and Theorem 2, one gets

$$
\left\{\begin{array}{l}
\frac{h}{2}\left(y\left(\varepsilon_{0}\right)-y\left(\eta_{0}\right)\right)^{2} \leq \frac{M^{2} h}{2}\left|\varepsilon_{0}-\eta_{0}\right| \leq \frac{M^{2} h^{3}}{8}, i=0, \\
h\left(y\left(\varepsilon_{i}\right)-y\left(\eta_{i}\right)\right)^{2} \leq M^{2} h\left|\varepsilon_{0}-\eta_{0}\right| \leq M^{2} h^{3}, 1 \leq i \leq m-1, \\
\frac{h}{2}\left(y\left(\varepsilon_{m}\right)-y\left(\eta_{m}\right)\right)^{2} \leq \frac{M^{2} h}{2}\left|\varepsilon_{m}-\eta_{m}\right| \leq \frac{M^{2} h^{3}}{8}, i=m .
\end{array}\right.
$$

We have

$$
\left\|e_{m}(x)\right\|^{2}=\int_{0}^{1} e_{m}^{2}(x) d x=\int_{0}^{1}\left(\sum_{i=0}^{m} e_{m, i}(x)_{i}\right)^{2} d x
$$

$=\int_{0}^{1}\left(\sum_{i=0}^{m} e_{m, i}(x)_{i}\right) d x+2 \sum_{i \leq j}^{m} \int_{0}^{1}\left(e_{m, i}(x) e_{m, j}(x)\right) d x d x$

Since for $i \neq j, I_{i} \cap I_{j}=\phi$, then

$\left\|e_{m}(x)\right\|^{2}=\int_{0}^{1}\left(\sum_{i=0}^{m} e_{m, i}^{2}(x)_{i}\right) d x=\sum_{i=0}^{m}\left\|e_{m, i}(x)\right\|^{2} d x$.

Afterward, one gets

$$
\left\|e_{m}(x)\right\|^{2} \leq M^{2} h^{2}-\frac{3 M^{2} h^{3}}{4},
$$

which completes the proof.

Suppose that $e_{m}^{\prime}(x)$ is the error between $y(x)$ and its BPFs expansion. As in Ref.[13], it is clear that

$$
\left\|e_{m}(x)\right\| \leq\left\|e_{m}^{\prime}(x)\right\|
$$

\section{Lemma 1}

Let $f(x)$ be defined as in Eq. (24), $g_{m}(x)$ be the IBPFs expansion approximation of $g(x)$ and $e_{g}(x)=g(x)-$ $g_{m}(x)$. Then

$$
\left\|e_{g}(x)\right\|=O(h)
$$

Proof

From Eq. (83), we have

$$
\left\|e_{g}(x)\right\|=\left(\sum_{i=1}^{m}\left\|g_{i}(x)-g_{m, i}(x)\right\|^{2}\right)^{1 / 2},
$$

and from Theorem 2, $\left\|g_{i}(x)-g_{m, i}(x)\right\|^{2} \leq C_{i} h$. Then

$$
\left\|e_{g}(x)\right\| \leq\left(\sum_{i=1}^{m} C_{i}^{2} h^{2}\right)^{1 / 2}=\left(\sum_{i=1}^{m} C_{i}^{2}\right)^{1 / 2} h=C h,
$$

which completes the proof.

Theorem 4

Let $k_{m}(x, y)$ be the IBPFs expansion approximation of $k(x, y)$ defined as in Eq. (7) and $k(x, y)$ be differentiable on $D \times D$ such that $\left|k^{\prime}(x, y)\right| \leq M$. Also, assume that $e_{m}(x, y)=k(x, y)-k_{m}(x, y)$, then

$$
\left\|e_{m}(x, y)\right\|=O(h) .
$$

Proof

Suppose $x_{0}=y_{0}=0, x_{i}=y_{i}=i h-\frac{h}{2}, i=1, \ldots, m$ and $x_{m+1}=y_{m+1}=1$. The error is defined between $k(x, y)$ and its IBPFs expansion over every subinterval $I_{i, i}=\left[x_{i}, x_{i+1}\right) \times$ $\left[y_{i}, y_{i+1}\right)$ as follows:

$$
e_{m, i j}(x, y)=k(x, y)-k_{i j}(x, y), x \in I_{i, j}, i, j=0,1, \ldots, m .
$$

By using the mean value theorem for integral and similar to the proof of Theorem 3, we get

$$
\left\|e_{m, i j}(x, y)\right\|^{2}=\left\{\begin{array}{l}
\frac{M^{2} h^{4}}{8}, i=0, m, \\
\frac{5 M^{2} h^{4}}{8}, 1 \leq i \leq m-1,
\end{array}\right.
$$

for $i=0, n$ and 


$$
\left\|e_{m, i j}(x, y)\right\|^{2}=\left\{\begin{array}{l}
\frac{5 M^{2} h^{4}}{8}, i=0, m, \\
2 M^{2} h^{4}, 1 \leq i \leq m-1,
\end{array}\right.
$$

for $i=1,2, \ldots, n-1$. We have

$$
\begin{aligned}
& \left\|e_{m}(x, y)\right\|^{2}=\int_{0}^{1} \int_{0}^{1} e_{m}^{2}(x, y) d x d y \\
& =\int_{0}^{1} \int_{0}^{1}\left(\sum_{i=0}^{m} \sum_{i=0}^{m} e_{m, i j}(x, y)_{i}\right)^{2} d x d y \\
& 2 \sum_{i \leq k}^{m} \sum_{i \leq l}^{m} \int_{0}^{1} \int_{0}^{1} e_{m, i j}(x, y) e_{m, k l}(x, y) d x d y . \text { (99) }
\end{aligned}
$$

Since for $i \neq k$ and $j \neq l$, we have $I_{i} \cap I_{k}=\phi$ and $I_{j} \cap I_{l}=\phi$, then

$$
\begin{gathered}
\left\|e_{m}(x, y)\right\|^{2}=\int_{0}^{1} \int_{0}^{1}\left(\sum_{i=0}^{m} \sum_{j=0}^{m} e_{m, i j}^{2}(x, y)\right) d x d y \\
=\sum_{i=0}^{m} \sum_{j=0}^{m}\left\|e_{m, i j}(x, y)\right\|^{2}
\end{gathered}
$$

Directly, we get

$$
\left\|e_{m}(x, y)\right\|^{2} \leq 2 M^{2} h^{2}-\frac{3 M^{2} h^{3}}{2} .
$$

Suppose $e_{n}^{\prime}(x, y)$ be the error between $k(x, y)$ and its BPFs expansion. From the work of Maleknejad et. al., [15], it is clear that

$$
\left\|e_{m}(x, y)\right\| \leq\left\|e_{m}^{\prime}(x, y)\right\|
$$

\section{Lemma 2}

Let $k(x, y)$ be as defined in Eq. $(27), k_{n}(x, y)$ be the IBPFs of $k(x, y)$ and

$$
\begin{array}{r}
e_{k}(x, y)=k(x, y)-k_{n}(x, y) \text {. Then } \\
\left\|e_{k}(x, y)\right\|=O(h) .
\end{array}
$$

Proof

From Eq. (84), we have

$\left\|e_{k}(x, y)\right\|=\left(\sum_{i=1}^{m} \sum_{j=1}^{m}\left\|k_{i j}(x, y)-k_{n, i j}(x, y)\right\|\right)^{1 / 2}$

From Theorem 3, we conclude that $\| k_{i j}(x, y)-$ $k_{n, i j}(x, y) \| \leq C_{i j}^{2} h$.

Therefore

$\left\|e_{k}(x, y)\right\| \leq\left(\sum_{i=0}^{m} \sum_{j=0}^{m} C_{i j}^{2} h^{2}\right)^{\frac{1}{2}}=\left(\sum_{i=0}^{m} \sum_{j=0}^{m} C_{i j}^{2}\right)^{\frac{1}{2}} h=C h$

Let the error of $M B P F \mathrm{~s}$ be denoted by

$$
E_{n}=\left\|y(x)-y_{n}(x)\right\|, x \in D,
$$

where $y(x)$ was defined in Eq. (22). Furthermore, assume the following hypotheses:

(M1) Let $\|y(x)\| \leq N$ for $x \in D$

(M2) Let $\|k(x, t)\| \leq N^{\prime}$ for $(x, t) \in D \times D$

(M3) According to lemma 1 and 2, let

$$
E_{g}=\left\|e_{g}(x)\right\| \leq C h,
$$

and

$$
E_{k}=\left\|e_{k}(x, t)\right\| \leq C^{\prime} h,
$$

where $C$ and $C^{\prime}$ are coefficients defined in Eqs. (95) and (105) and $g(x)$ and $k(x, t)$ were defined in Eqs. (26) and (25), respectively:

$$
\text { (M4) Let } N^{\prime}+C^{\prime} h<1 \text {. }
$$

\section{Theorem 5}

Let $y(x)$ and $y_{n}(x)$ be the exact and approximate solutions of Eq. (1) or Eq. (2) respectively. Also, the assumptions (M1)-(M4) are satisfied.

Therefore, we have

$$
E_{n} \leq \frac{\left(C+C^{\prime} N\right) h}{1-N^{\prime}-C h}
$$

Proof

For the first case, from Eq. (2), we have

$$
\begin{aligned}
y(x)-y_{n}(x)= & g(x)-g_{n}(x)+\int_{0}^{x}(k(x, t) y(t)- \\
& \left.k_{n}(x, t) y_{n}(t)\right) d t,
\end{aligned}
$$

and therefore

$$
E_{n} \leq E_{g}+\|x\|\left\|k(x, t) y(t)-k_{n}(x, t) y_{n}(t)\right\| .
$$

It is clear that $\|x\| \leq 1$, So

$$
E_{n} \leq E_{g}+\left\|k(x, t) y(t)-k_{n}(x, t) y_{n}(t)\right\|
$$

Also, for the second case, from Eq. (1), we have

$$
\begin{aligned}
y(x)-y_{n}(x)= & g(x)-g_{n}(x)+\int_{0}^{1}(k(x, t) y(t)- \\
& \left.k_{n}(x, t) y_{n}(t)\right) d t,
\end{aligned}
$$

and therefore

$$
E_{n} \leq E_{g}+\left\|k(x, t) y(t)-k_{n}(x, t) y_{n}(t)\right\| .
$$

So, Eq. (98) is true in both cases.

Now, according to assumptions (M1)-(M3), we have

$$
\begin{array}{r}
\left\|k(x, t) y(t)-k_{n}(x, t) y_{n}(t)\right\| \leq\|k(x, t)\| E_{n}+ \\
E_{k}\left(E_{n}+\|y(x)\|\right) \leq N^{\prime} E_{n}+C^{\prime} h\left(E_{n}+N\right) .
\end{array}
$$

Also, from assumption (M3), we have

$$
E_{n} \leq\left(C+C^{\prime} N\right) h+\left(N^{\prime}+C^{\prime} h\right) E_{n} .
$$

Therefore according to (M4), this equation is satisfied and this completes the proof.

Also, we have $E_{n}=O(h)$. 


\section{Lemma 3}

Suppose that the functions $y(x)$ and $y_{n}(x)$ are the exact and approximate solution of Eq. (2) or Eq. (1), respectively, where $y(x)$ was defined in Eq. (22) and

$$
y_{n}(x)=\left[y_{1, n}(x), y_{2, n}(x), \ldots, y_{m, n}(x)\right]^{T}
$$

Then

$$
e_{i, n}=\left\|y_{i}(x)-y_{i, n}(x)\right\|=O(h)
$$

Proof

From Theorem 4, we have $E_{n} \leq C h$ and according to Eq. (83), one gets

$$
e_{i, n} \leq E_{n} \leq C h \text {. }
$$

The series solution $y_{n}(x)$ will be the approximate solution of Eq. (2) or Eq. (1), where the function $y(x)$ is defined in Eq. (22) and

$$
y_{m}(x)=\sum_{m=1}^{i} y_{i} \phi_{i}(x),
$$

converges to the exact solution $y(x)$ then

$$
\lim _{m \rightarrow \infty}\left\|y(x)-y_{m}(x)\right\|^{2}=0 .
$$

\section{Theorem 6}

Let $L^{2}(\mathbb{R})$ be Helbert space and $\phi_{i}(x)$ defined in Eq. (13) form a basis of IBPFs. (1).

Let $y(x) \simeq \sum_{m=1}^{i} y_{i} \phi_{i}(x)$ be the solution of Eq. (2) or Eq.

Now, we define a sequence of partial sums $S_{i}$ of $\left(\alpha_{m} \phi_{i}(x)\right)$. Let $S_{i}$ and $S_{j}$ be the partial sums with $i \geq j$. We have to prove $S_{i}$ is a Cauchy sequence in the Hilbert space.

Proof

$$
\text { Let } S_{i}=\sum_{m=1}^{i} \alpha_{m} \phi_{i}(x)
$$

Now,

$$
\begin{array}{r}
<y(x), S_{i} \geq<y(x), \sum_{m=1}^{i} \alpha_{m} \phi_{i}(x) \geq \sum_{m=1}^{i} \alpha_{m}< \\
y(x), \phi_{i}(x)=\sum_{m=1}^{i} \alpha_{m} \alpha_{m}=\sum_{m=1}^{i}\left|\alpha_{m}\right|^{2} .(122)
\end{array}
$$

We claim that

$$
\begin{gathered}
\left\|S_{i}-S_{j}\right\|^{2}=\left\|\sum_{m=1}^{i} \alpha_{m} \phi_{i}(x)\right\|^{2}= \\
<\sum_{m=j+1}^{i} \alpha_{m} \phi_{i}(x), \sum_{m=j+1}^{i} \alpha_{m} \phi_{i}(x)> \\
=\sum_{m=j+1}^{i} \sum_{m=j+1}^{i} \alpha_{m} \alpha_{m}<\phi_{i}(x), \phi_{i}(x)>= \\
\sum_{m=j+1}^{i}\left|\alpha_{m}\right|^{2} .
\end{gathered}
$$

Therefore,

$$
\begin{array}{r}
\left\|\sum_{m=j+1}^{i} \alpha_{m} \phi_{i}(x) \alpha_{m} \phi_{i}(x)\right\|^{2}= \\
\sum_{m=j+1}^{i} \alpha_{m} \phi_{i}(x)\left|\alpha_{m}\right|^{2}, \text { fori }>j .
\end{array}
$$

From Bessel's inequality, we have

$$
\sum_{m=j+1}^{i} \alpha_{m} \phi_{i}(x)\left|\alpha_{m}\right|^{2}
$$

It is convergent and hence

$$
\left\|\sum_{m=j+1}^{i} \alpha_{m} \phi_{i}(x)\right\|^{2} \rightarrow 0, a s i, j \rightarrow \infty .
$$

Hence, we have

$$
\left\|\sum_{m=j+1}^{i} \alpha_{m} \phi_{i}(x)\right\| \rightarrow 0,
$$

and $S_{i}$ is a Cauchy sequence and it converges to $s$ (say). We assert that $y(x)=s$.

Now,

$$
\begin{gathered}
<s-y(x), \phi_{i}(x) \geq<s, \phi_{i}(x)><y(x), \phi_{i}(x) \geq \\
<\lim _{m \rightarrow \infty} S_{i}, \phi_{i}(x)>\phi_{i}(x)=\lim _{m \rightarrow \infty}<\alpha_{m} \phi_{i}(x), \phi_{i}(x)> \\
\phi_{i}(x)=\phi_{i}(x)-\phi_{i}(x)=0 .
\end{gathered}
$$

We conclude that

$$
<s-y(x), \phi_{i}(x) \geq 0 .
$$

Hence $y(x)=s$ and $S_{i}=\sum_{m=1}^{i} \alpha_{m} \phi_{i}(x)$ converges to $y(x)$ as $i \rightarrow \infty$ and proved.

The above relation is possible if

$$
u(x)=s .
$$

\section{Numerical Modeling}

This part included some physical models that will be solved by using the current improved technique to demonstrate the reliability and efficiency of these modifications. Furthermore, it includes numerical comparisons between the present method and other similar methods in the algorithm, to show the accuracy of each of them. Some figures and tables might be included in each model for clarification. All methods used in these comparisons are used by many authors to solve many problems.

\section{Example 1}

We will start with a Fredholm type integral equations [16]

$$
y(t)=e^{2 t+\frac{1}{3}}-\frac{1}{3} \int_{0}^{1} e^{2 t-\left(\frac{5}{3}\right) s} y(s) d s,
$$

where the exact solution is $y(t)=e^{2 t}$.

Suppose

$$
\begin{gathered}
y(t) \simeq Y^{T} \Psi_{(m)}(t) \simeq \Psi_{(m)}^{T}(t) Y \\
e^{2 t+\frac{1}{3}} \simeq F^{T} \Psi_{(m)}(t) \\
e^{2 t-\left(\frac{5}{3}\right) s} \simeq \Psi_{(m)}^{T}(s) K_{(m)} \Psi_{(m)}(t)
\end{gathered}
$$

where $Y^{T}=\left[y_{0}, y_{1}, \ldots, y_{m}\right]$ are the undetermined coefficients for the unknown function $y(t), F^{T}=\left[f_{0}, f_{1}, \ldots, f_{m}\right]$ are known and is found by using 


$$
f_{i}=\left\{\begin{array}{l}
2 m \int_{0}^{\frac{i+1 / 2}{m}} e^{2 t+\frac{1}{3}} d t, i=0 \\
m \int_{\frac{i-1 / 2}{m}}^{1 / 2 m} e^{2 t+\frac{1}{3}} d t, 1 \leq i \leq m-1, \\
2 m \int_{1-1 / 2 \mathrm{~m}}^{1} e^{2 t+\frac{1}{3}} d t, i=m .
\end{array}\right.
$$

Also, $K_{(m)}=K_{m+1 \times m+1}$ can be found by using the following relation:

$$
K_{(m)} \simeq\left[k_{i j}\right]_{(m+1 \times m+1)}
$$

Substituting in Eq. (131)

$$
\begin{gathered}
Y^{T} \Psi_{(m)}(t) \simeq \\
F^{T} \Psi_{(m)}(t)-\frac{1}{3} \int_{0}^{1} \Psi_{(m)}^{T}(s) K_{(m)} \Psi_{(m)}(t) \Psi_{(m)}^{T}(t) Y .
\end{gathered}
$$

From Eq. (32), one gets

$$
Y^{T}=F^{T}\left[I_{(m+1)}-\frac{1}{3} K_{(m)} V\right]^{-1} .
$$

By solving this system of linear equations, the improved block pulse series coefficients can be found. After substituting into Eq. (131), the IBPF approximate solution will be found. Below are the graphs of the improved block pulse approximate solutions at $m=32$. Also, the exact solution is graphed on the same axes to see how close is the new method to the exact solution in the selected intervals. The points here are taken as the midpoints of the intervals of the IBPFs that is why the graph of the exact solution absolutely coincides with the graph of the IBPFs.

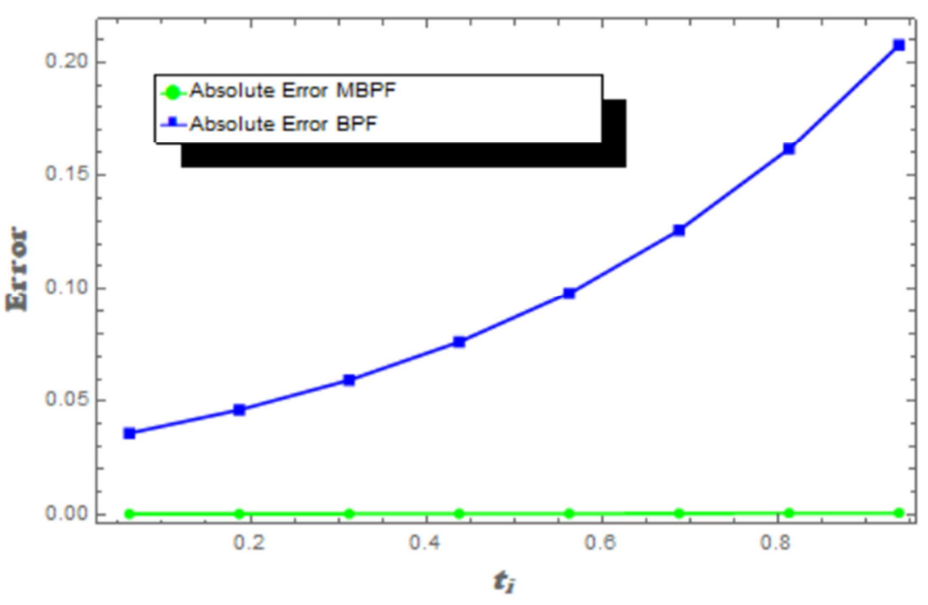

Figure 1. Absolute error of IBPFs and BPFs expansions. A comparison at the midpoints of the intervals of IBPFs was done.

One can notices that the error is huge in this case. However; if we choose random points the functions will be like

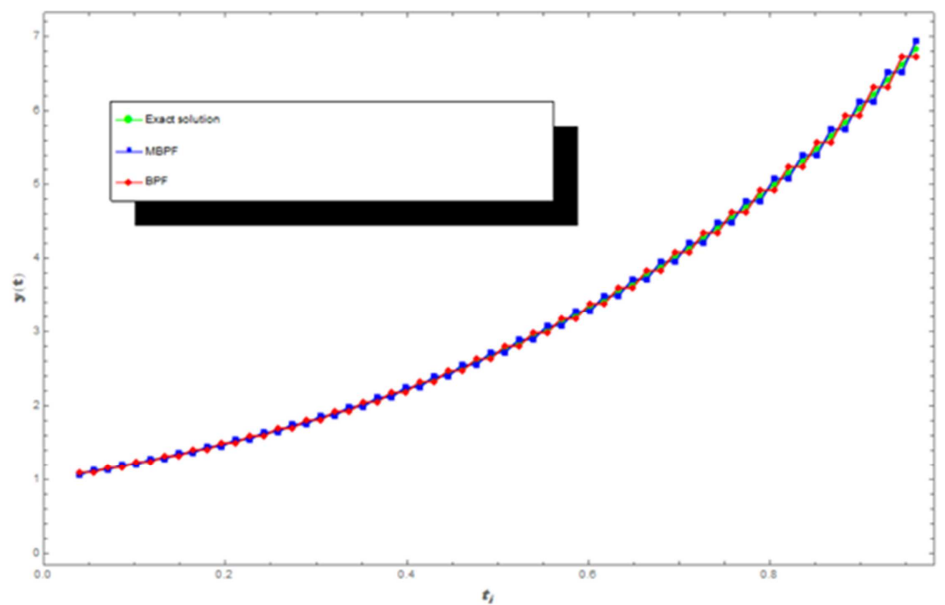

Figure 2. A comparison between the exact and approximation of both BPFs and IBPFs solutions.

It is noticed here that at some points the BPFs solution is better and at other points, the IBPFs solution is better. The error, in this case, will be sometimes in the favor of IBPFs and at other points will be in the favor of BPFs as. 


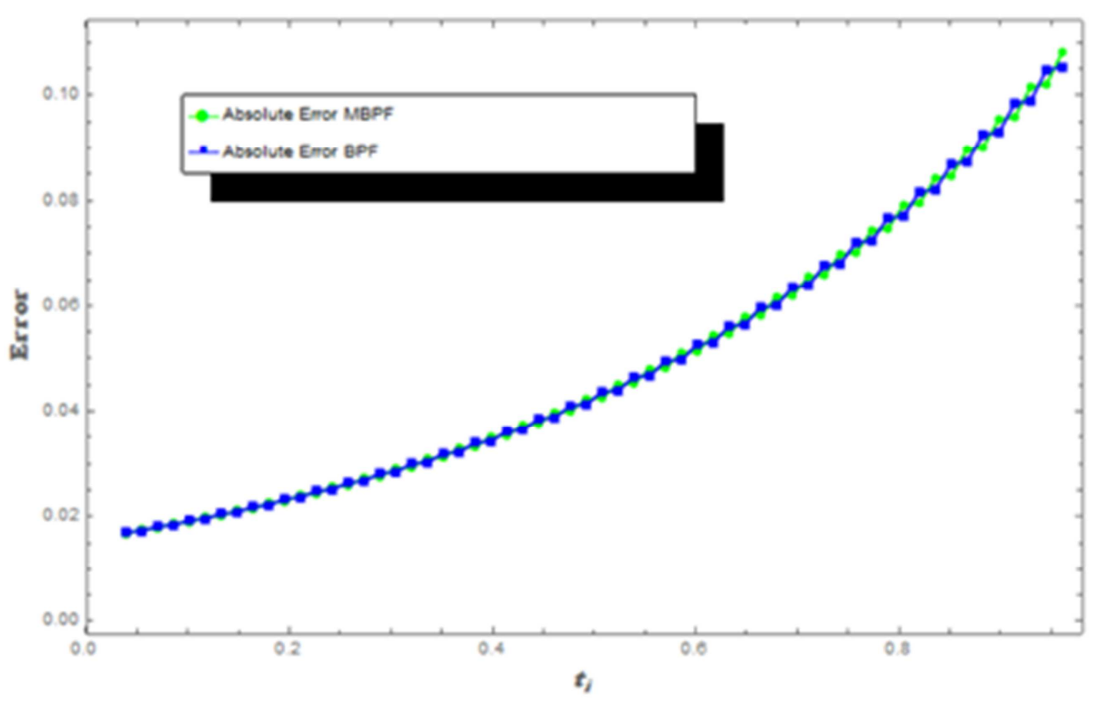

Figure 3. Absolute error of IBPFs and BPFs expansions. A comparison at random points is done.

The following table shows numerical results of the absolute error by using the block pulse method and the improved block pulse method at the midpoint of every subinterval. Also, the error for the present method is compared with the block pulse functions error at some points in the following table at $m=16$.

Table 1. The numerical results of example 1 at $m=16$.

\begin{tabular}{llllll}
\hline $\mathbf{t}$ & \multirow{2}{*}{ Exact solution } & IBPF & \multirow{2}{*}{ BPF } & Absolute error \\
\cline { 5 - 6 } & & & IBPF & BPF \\
\hline 0.0625 & 1.133148453 & 1.1335535982 & 1.2066444069 & $4.05145 \times 10^{-4}$ & $7.3496 \times 10^{-2}$ \\
0.1875 & 1.45499141461 & 1.4555116313 & 1.5493620874 & $5.20217 \times 10^{-4}$ & $9.43707 \times 10^{-2}$ \\
0.3125 & 1.86824595743 & 1.86891392893 & 1.9894202999 & $6.67972 \times 10^{-4}$ & $1.21174 \times 10^{-1}$ \\
0.4375 & 2.39887529396 & 2.3997329863 & 2.5544662295 & $8.57692 \times 10^{-4}$ & $1.55591 \times 10^{-1}$ \\
0.5625 & 3.0802168489 & 3.08131814774 & 3.2799995648 & $1.1013 \times 10^{-3}$ & $1.99783 \times 10^{-1}$ \\
0.6875 & 3.9550767229 & 3.95649081860 & 4.211602808 & $1.4141 \times 10^{-3}$ & $2.56526 \times 10^{-1}$ \\
0.8125 & 5.07841903718 & 5.0802347720 & 5.4078050504 & $1.81573 \times 10^{-3}$ & $3.29386 \times 10^{-1}$ \\
0.9375 & 6.52081912033 & 6.5228219472 & 6.943759133 & $2.00283 \times 10^{-3}$ & $4.2294 \times 10^{-1}$ \\
\hline
\end{tabular}

For $\mathrm{m}=32$ we will find the following output

Table 2. The numerical results of example 1 at $m=32$.

\begin{tabular}{|c|c|c|c|c|c|}
\hline \multirow{2}{*}{$\mathbf{t}$} & \multirow{2}{*}{ Exact solution } & \multirow{2}{*}{ IBPF } & \multirow{2}{*}{ BPF } & \multicolumn{2}{|c|}{ Absolute error } \\
\hline & & & & IBPF & BPF \\
\hline 0.0625 & 1.1331484530668 & 1.1332478037112 & 1.1692188240521 & $9.93506 \times 10^{-5}$ & $3.60704 \times 10^{-2}$ \\
\hline 0.1875 & 1.4549914146182 & 1.4551189833707 & 1.5013066877527 & $1.27569 \times 10^{-4}$ & $4.63153 \times 10^{-2}$ \\
\hline 0.3125 & 1.8682459574322 & 1.8684097589528 & 1.9277159453177 & $1.63802 \times 10^{-4}$ & $5.947 \times 10^{-2}$ \\
\hline 0.4375 & 2.398875293967 & 2.3990856192828 & 2.4752362699422 & $2.10325 \times 10^{-4}$ & $7.6361 \times 10^{-2}$ \\
\hline 0.5625 & 3.08021684891 & 3.0804869119692 & 3.1782662829132 & $2.70063 \times 10^{-4}$ & $9.80494 \times 10^{-2}$ \\
\hline 0.6875 & 3.955076722920 & 3.9554234907425 & 4.0809746882622 & $3.46768 \times 10^{-4}$ & $1.25898 \times 10^{-1}$ \\
\hline 0.8125 & 5.0784190371800 & 5.07886429587 & 5.2400752245881 & $4.45259 \times 10^{-4}$ & $1.61656 \times 10^{-1}$ \\
\hline 0.9375 & 6.5208191203301 & 6.521390843816 & 6.728389773726 & $5.71723 \times 10^{-4}$ & $2.07571 \times 10^{-1}$ \\
\hline
\end{tabular}

It is worth noting here is that the midpoint of the intervals is different for each method as each method has different intervals. In the study of Maleknejad, and Mahmoudi, [16], the authors used the hybrid Taylor and block-pulse functions. They got the maximum norm of the error. We notice that at $\mathrm{m}=32$, the IBPFs maximum norm of error is $5.71723 \times$ $10^{-4}$ which is less error and a more accurate solution than that done by using the hybrid Taylor and block-pulse functions. In the study of Maleknejad, and Mahmoudi, [16], the table done is as follows:
Table 3. The numerical results as done by using hybrid Taylor and block-pulse functions [16].

\begin{tabular}{llll}
\hline $\mathbf{M}$ & $\mathbf{N}$ & $\left\|\boldsymbol{y}-\boldsymbol{y}^{\boldsymbol{B}}\right\|_{\infty}$ & Cond (I - KD) \\
\hline 3 & 10 & $3.005651 \times 10^{-2}$ & 6.0920 \\
3 & 20 & $8.668870 \times 10^{-3}$ & 7.0734 \\
3 & 40 & $2.316608 \times 10^{-3}$ & 7.6292 \\
3 & 80 & $5.981580 \times 10^{-4}$ & 7.9248 \\
4 & 10 & $2.892984 \times 10^{-2}$ & 7.1086 \\
4 & 20 & $7.351252 \times 10^{-3}$ & 8.3168 \\
4 & 40 & $1.847061 \times 10^{-3}$ & 9.0048 \\
4 & 80 & $4.625381 \times 10^{-4}$ & 9.3718 \\
\hline
\end{tabular}




\section{Example 2}

Now, we will study a Volterra numerical example to show how the method works on it [17],

$$
y(t)=\operatorname{Cos}(t)+\int_{0}^{t}(t-s) \operatorname{Cos}(t-s) y(s) d s
$$

where the exact solution is $y(t)=\frac{1}{3}(2 \operatorname{Cos}(\sqrt{3} t+1))$.

Let

$$
\begin{gathered}
y(t) \simeq Y^{T} \Psi_{(m)}(t) \\
\operatorname{Cos}(t) \simeq F^{T} \Psi_{(m)}(t) \\
(t-s) \operatorname{Cos}(t-s) \simeq \Psi_{(m)}^{T}(s) K_{(m)} \Psi_{(m)}(t)
\end{gathered}
$$

where $Y^{T}=\left[y_{0}, y_{1}, \ldots, y_{m}\right]$ are the undetermined coefficients for the unknown function $y(t), F^{T}=$ $\left[f_{0}, f_{1}, \ldots, f_{m}\right]$ are known and found by using

$$
f_{i}=\left\{\begin{array}{l}
2 m \int_{0}^{1 / 2 m} \operatorname{Cos}(t) d t, i=0 \\
m \int_{\frac{i-1 / 2}{m}}^{\frac{i+1 / 2}{m}} \operatorname{Cos}(t) d t, 1 \leq i \leq m-1, \\
2 m \int_{1-1 / 2 \mathrm{~m}}^{1} \operatorname{Cos}(t) d t, i=m .
\end{array}\right.
$$

Also, $K_{(m)}=K_{m+1 \times m+1}$ can be found by using the following relation:

$$
K_{(m)}=\left[k_{i j}\right]_{(m+1 \times m+1)}
$$

Substituting in Eq. (132), one gets

$$
\begin{aligned}
& Y^{T} \Psi_{(m)}(t)=F^{T} \Psi_{(m)}(t) \\
& +\int_{0}^{t} \Psi_{(m)}^{T}(s) K_{(m)} \Psi_{(m)}(t) \Psi_{(m)}^{T}(t) Y .
\end{aligned}
$$

From Eq. (32), one finds

$$
Y^{T}=F^{T}\left[I_{(m+1)}-K_{(m)} B\right]^{-1} .
$$

Then by solving this system of linear equations, the improved block pulse series coefficients can be found. After substituting into Eq. (132), the IBPF approximate solution will be found. Below are the graphs of the improved block pulse approximate solutions at $m=32$. Also, the exact solution is graphed on the same axes to see how close is the new method to the exact solution in the selected intervals. We notice here that as we increase the number of intervals, the MPBF coincides with the exact solution. The graphs of the block pulse function solution at the same divisions of intervals are also graphed. Now, we can look at the combined graphs of the $\mathrm{BPF}, \mathrm{IBPF}$, and exact solutions at $\mathrm{m}=32$.

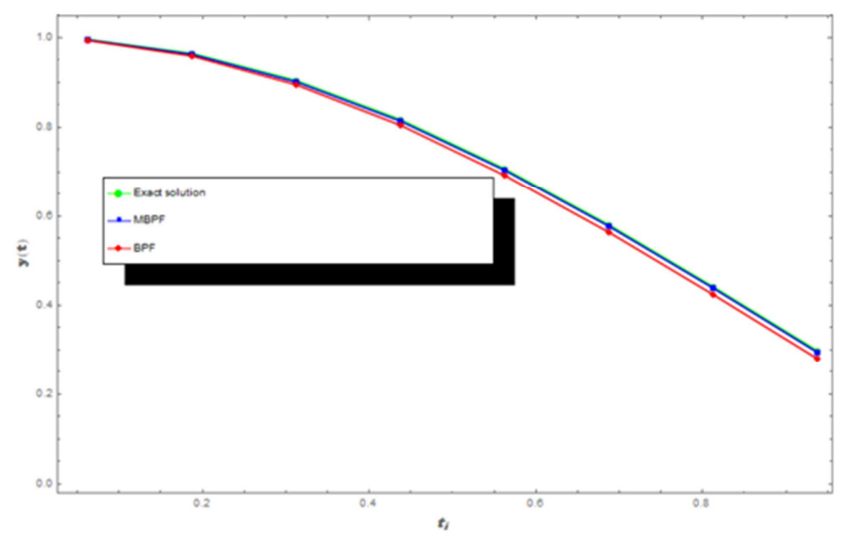

Figure 4. Absolute error of IBPFs and BPFs expansions. A comparison at the midpoints of the intervals of IBPFs was done.

The error, in this case, will be like this

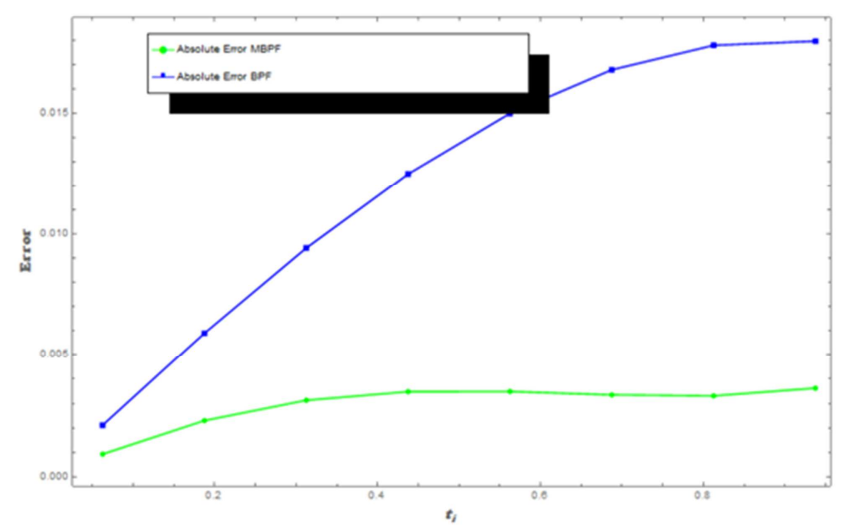

Figure 5. Absolute error of IBPFs and BPFs expansions. A comparison at the midpoints of the intervals of IBPFsis was done.

One notices that the error is huge in this case. However; if we choose random points the functions will be like this

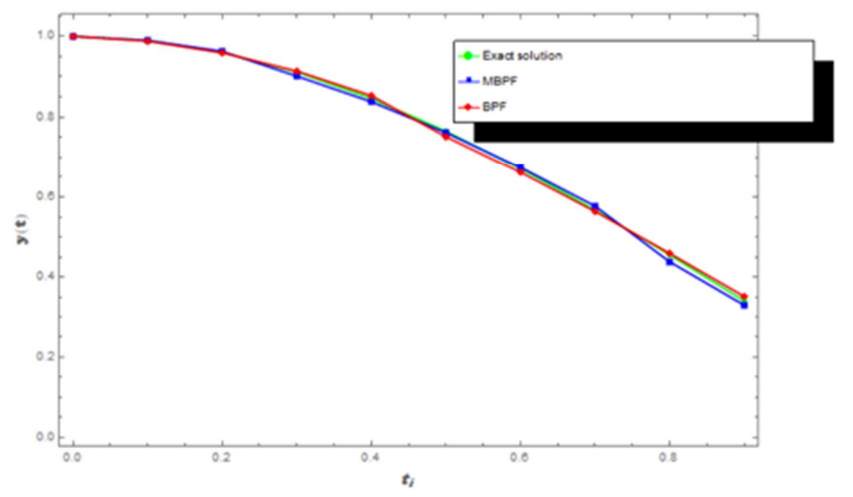

Figure 6. A comparison between the exact and the approximation of both $B P F$ s and IBPFs solutions.

We notice here that at some points the BPFs solution is better and at other points, the IBPFs solution is better. The error in this case also will be sometimes in the favor of IBPFs and at other points will be in the favor of BPFs as follows.

Now the following tables show the values of the exact, BPF, and IBPF solutions at different points within the interval $[0,1)$. Notice that the modification done to the BPF made the 
absolute error smaller than the regular BPF. Also, it's worth mentioning that it took less time to compute the solution using

IBPF than BPF.

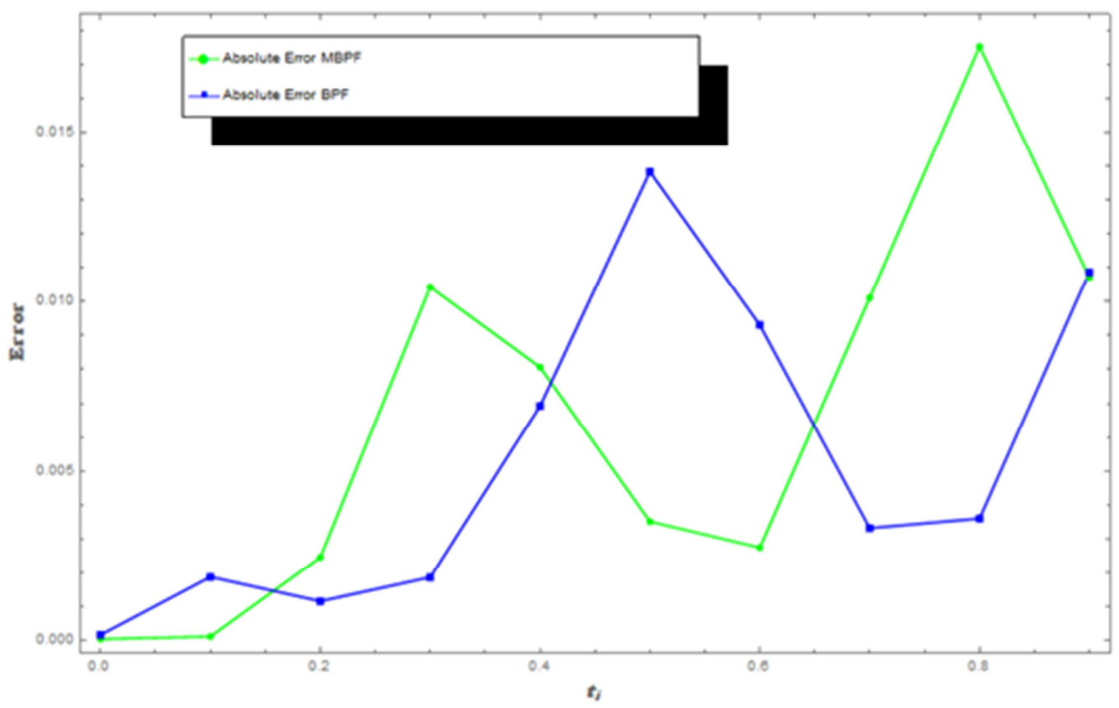

Figure 7. Absolute error of IBPFs and BPFs expansions. A comparison at random points is done.

Table 4. The numerical results of example 3 at $m=8,16$ and 32 .

\begin{tabular}{|c|c|c|c|c|c|}
\hline \multirow{2}{*}{$\mathbf{t}$} & \multirow{2}{*}{ Exact solution } & \multirow{2}{*}{ IBPF } & \multirow{2}{*}{ BPF } & \multicolumn{2}{|l|}{ Absolute error } \\
\hline & & & & IBPF & BPF \\
\hline 0.0625 & 0.99609757 & 0.99739787 & 0.97780024 & $1.3003 \times 10^{-3}$ & $1.82973 \times 10^{-2}$ \\
\hline 0.1875 & 0.96515166 & 0.96643162 & 1.90563427 & $1.2800 \times 10^{-3}$ & $9.404826 \times 10^{-1}$ \\
\hline 0.3125 & 0.90470478 & 0.90594438 & 1.77933478 & $1.2396 \times 10^{-3}$ & $8.746300 \times 10^{-1}$ \\
\hline 0.4375 & 0.81757932 & 0.81875923 & 1.60339295 & $1.1799 \times 10^{-3}$ & $7.858136 \times 10^{-1}$ \\
\hline 0.5625 & 0.70784335 & 0.70894532 & 1.38491319 & $1.1020 \times 10^{-3}$ & $6.770698 \times 10^{-1}$ \\
\hline 0.6875 & 0.5806207 & 0.58162799 & 1.13268493 & $1.0073 \times 10^{-3}$ & $5.520642 \times 10^{-1}$ \\
\hline 0.8125 & 0.44185167 & 0.44274958 & 0.85683929 & $8.979 \times 10^{-4}$ & $4.149876 \times 10^{-1}$ \\
\hline 0.9375 & 0.29801567 & 0.29879217 & 0.60443488 & $7.765 \times 10^{-4}$ & $3.064192 \times 10^{-1}$ \\
\hline \multirow{2}{*}{$\mathbf{t}$} & \multirow{2}{*}{ Exact solution } & \multirow{2}{*}{ IBPF } & \multirow{2}{*}{ BPF } & \multicolumn{2}{|l|}{ Absolute error } \\
\hline & & & & IBPF & BPF \\
\hline 0.03125 & 0.99902368 & 0.99934909 & 0.99421874 & $3.254 \times 10^{-4}$ & $4.8049 \times 10^{-3}$ \\
\hline 0.15625 & 0.97573459 & 0.97605619 & 1.94171116 & $3.216 \times 10^{-4}$ & $9.659766 \times 10^{-1}$ \\
\hline 0.28125 & 0.92245038 & 0.92276315 & 1.83156224 & $3.128 \times 10^{-4}$ & $9.091119 \times 10^{-1}$ \\
\hline 0.40625 & 0.84165902 & 0.84195808 & 1.66841447 & $2.991 \times 10^{-4}$ & $8.267555 \times 10^{-1}$ \\
\hline 0.53125 & 0.73713282 & 0.73741355 & 1.45933925 & $2.807 \times 10^{-4}$ & $7.222064 \times 10^{-1}$ \\
\hline 0.65625 & 0.61375234 & 0.61401045 & 1.21337334 & $2.581 \times 10^{-4}$ & $5.996210 \times 10^{-1}$ \\
\hline 0.78125 & 0.47727849 & 0.47751017 & 0.94113432 & $2.317 \times 10^{-4}$ & $4.638558 \times 10^{-1}$ \\
\hline 0.90625 & 0.33408352 & 0.3342856 & 0.65436891 & $2.021 \times 10^{-4}$ & $3.202854 \times 10^{-1}$ \\
\hline
\end{tabular}

\begin{tabular}{|c|c|c|c|c|c|}
\hline \multirow{2}{*}{$\mathbf{t}$} & \multirow{2}{*}{ Exact solution } & \multirow{2}{*}{ IBPF } & \multirow{2}{*}{ BPF } & \multicolumn{2}{|c|}{ Absolute error } \\
\hline & & & & IBPF & BPF \\
\hline 0.015625 & 0.99975588 & 0.99983725 & 0.99852481 & $8.14 \times 10^{-5}$ & $1.2311 \times 10^{-3}$ \\
\hline 0.140625 & 0.98032218 & 0.98040276 & 1.95648495 & $8.06 \times 10^{-5}$ & $9.761628 \times 10^{-1}$ \\
\hline 0.265625 & 0.93067917 & 0.9307577 & 1.85517284 & $7.85 \times 10^{-5}$ & $9.244937 \times 10^{-1}$ \\
\hline 0.390625 & 0.85314478 & 0.85322004 & 1.69912267 & $7.53 \times 10^{-5}$ & $8.459779 \times 10^{-1}$ \\
\hline 0.515625 & 0.75133926 & 0.75141007 & 1.49535215 & $7.08 \times 10^{-5}$ & $7.440129 \times 10^{-1}$ \\
\hline 0.640625 & 0.63001613 & 0.63008142 & 1.25300932 & $6.53 \times 10^{-5}$ & $6.229932 \times 10^{-1}$ \\
\hline 0.765625 & 0.49484024 & 0.49489903 & 0.98296517 & $5.88 \times 10^{-5}$ & $4.881249 \times 10^{-1}$ \\
\hline 0.890625 & 0.35212323 & 0.35217472 & 0.69732962 & $5.15 \times 10^{-5}$ & $3.452064 \times 10^{-1}$ \\
\hline
\end{tabular}

\section{Example 3}

Now, a non-linear Volterra integral equation will be considered [18],

$$
y(t)=\sin t+\frac{1}{8} \sin 2 t-\frac{1}{4} t+\int_{0}^{t} \frac{1}{2}(y(s))^{2} d s
$$

where the exact solution is $y(t)=\tan (t)$.

Let

$$
\begin{gathered}
y(t) \simeq Y^{T} \Psi_{(m)}(t) \\
\sin t+\frac{1}{8} \sin 2 t-\frac{1}{4} t \simeq F^{T} \Psi_{(m)}(t)
\end{gathered}
$$




$$
\begin{gathered}
\frac{1}{2} \simeq \Psi_{(m)}^{T}(s) K_{(m)} \Psi_{(m)}(t) \\
y(t)]^{2} \simeq \tilde{Y}^{T} \phi_{m}(t),
\end{gathered}
$$

where

$$
\tilde{Y}=\left[y_{0}^{2}, y_{1}^{2}, \cdots, y_{m-1}^{2}, y_{m}^{2}\right]
$$

and $Y^{T}=\left[y_{0}, y_{1}, \ldots, y_{m}\right]$ are the undetermined coefficients for the unknown function $y(t), F^{T}=\left[f_{0}, f_{1}, \ldots, f_{m}\right]$ are known and found by using

$$
f_{i}=\left\{\begin{array}{l}
2 m \int_{0}^{1 / 2 m} \sin t+\frac{1}{8} \sin 2 t-\frac{1}{4} t d t, i=0, \\
m \int_{\frac{i-1 / 2}{m}}^{\frac{i+1 / 2}{m}} \sin t+\frac{1}{8} \sin 2 t-\frac{1}{4} t d t, 1 \leq i \leq m-1, \\
2 m \int_{1-1 / 2 \mathrm{~m}}^{1} \sin t+\frac{1}{8} \sin 2 t-\frac{1}{4} t d t, i=m .
\end{array}\right.
$$

Also, $K_{(m)}=K_{m+1 \times m+1}$ can be found by using the following relation:

$$
K_{(m)} \simeq\left[k_{i j}\right]_{(m+1 \times m+1)}
$$

Substituting in Eq. (133), one gets

$$
Y^{T} \Psi_{(m)}(t)=F^{T} \Psi_{(m)}(t)+\int_{0}^{t} \Psi_{(m)}^{T}(s) K_{(m)} \Psi_{(m)}(t) \Psi_{(m)}^{T}(t) Y
$$

then

$$
Y_{m}=F_{m}+K B \tilde{Y},
$$

Then by solving this system of linear equations, the improved block pulse series coefficients can be found. After substituting into Eq. (133), the IBPF approximate solution will be found. Now, the following tables show the values of the

\begin{tabular}{|c|c|c|c|c|c|}
\hline \multirow{2}{*}{$\mathbf{t}$} & \multirow{2}{*}{ Exact solution } & \multirow{2}{*}{ BPF } & \multirow{2}{*}{ IBPF } & \multicolumn{2}{|l|}{ Absolute error } \\
\hline & & & & BPF & IBPF \\
\hline 0.0625 & 0.06245931784 & 0.093612675778 & 0.062466951295 & $3.11534 \times 10^{-2}$ & $7.63345 \times 10^{-6}$ \\
\hline 0.1875 & 0.18640329676 & 0.2170087570684 & 0.186410549346 & $3.06055 \times 10^{-2}$ & $7.25258 \times 10^{-6}$ \\
\hline 0.3125 & 0.307438514580 & 0.337016820351 & 0.307444087500 & $2.95783 \times 10^{-2}$ & $5.57292 \times 10^{-6}$ \\
\hline 0.4375 & 0.423676257203 & 0.451763228136 & 0.423677936691 & $2.8087 \times 10^{-2}$ & $1.67949 \times 10^{-6}$ \\
\hline 0.5625 & 0.53330267353 & 0.559456455022 & 0.533297375625 & $2.61538 \times 10^{-2}$ & $5.29791 \times 10^{-6}$ \\
\hline 0.6875 & 0.634607080015 & 0.658415051904 & 0.634590915940 & $2.3808 \times 10^{-2}$ & $1.61641 \times 10^{-5}$ \\
\hline 0.8125 & 0.726008655260 & 0.747093898134 & 0.725977021657 & $2.10852 \times 10^{-2}$ & $3.16336 \times 10^{-5}$ \\
\hline 0.9375 & 0.80608110826 & 0.824108333579 & 0.806028807496 & $1.80272 \times 10^{-2}$ & $5.23008 \times 10^{-5}$ \\
\hline \multirow{2}{*}{$\mathbf{t}$} & \multirow{2}{*}{ Exact solution } & \multirow{2}{*}{ BPF } & \multirow{2}{*}{ IBPF } & \multicolumn{2}{|l|}{ Absolute error } \\
\hline & & & & BPF & IBPF \\
\hline 0.03125 & 0.0312449139853 & 0.046857834016 & 0.031245867786 & $1.56129 \times 10^{-2}$ & $9.53801 \times 10^{-7}$ \\
\hline 0.15625 & 0.1556149927735 & 0.171029920177 & 0.155615881844 & $1.54149 \times 10^{-2}$ & $8.89071 \times 10^{-7}$ \\
\hline 0.28125 & 0.277556751646 & 0.292532814144 & 0.277557294759 & $1.49761 \times 10^{-2}$ & $5.43113 \times 10^{-7}$ \\
\hline 0.40625 & 0.3951673302409 & 0.409470265960 & 0.395167014811 & $1.43029 \times 10^{-2}$ & $3.15429 \times 10^{-7}$ \\
\hline 0.53125 & 0.506611454814 & 0.520017268808 & 0.506609546436 & $1.34058 \times 10^{-2}$ & $1.90838 \times 10^{-6}$ \\
\hline 0.65625 & 0.610150077075 & 0.622448539197 & 0.610145633976 & $1.22985 \times 10^{-2}$ & $4.4431 \times 10^{-6}$ \\
\hline 0.78125 & 0.704167511454 & 0.715165442270 & 0.704159404855 & $1.09979 \times 10^{-2}$ & $8.1066 \times 10^{-6}$ \\
\hline 0.90625 & 0.787196647331 & 0.796720942461 & 0.787183588996 & $9.5243 \times 10^{-3}$ & $1.30583 \times 10^{-5}$ \\
\hline \multirow{2}{*}{$\mathbf{t}$} & \multirow{2}{*}{ Exact solution } & \multirow{2}{*}{ BPF } & \multirow{2}{*}{ IBPF } & \multicolumn{2}{|l|}{ Absolute error } \\
\hline & & & & BPF & IBPF \\
\hline 0.015625 & 0.01562436422 & 0.023435354237 & 0.01562448343 & $7.81099 \times 10^{-3}$ & $1.19213 \times 10^{-7}$ \\
\hline 0.140625 & 0.14016197234 & 0.147892979326 & 0.14016207867 & $7.73101 \times 10^{-3}$ & $1.0633 \times 10^{-7}$ \\
\hline 0.265625 & 0.26251239976 & 0.270042713246 & 0.26251242836 & $7.53031 \times 10^{-3}$ & $2.85989 \times 10^{-8}$ \\
\hline 0.390625 & 0.38076640899 & 0.387978390767 & 0.38076623687 & $7.21198 \times 10^{-3}$ & $1.72115 \times 10^{-7}$ \\
\hline 0.515625 & 0.49307868575 & 0.499859605785 & 0.49307813398 & $6.78092 \times 10^{-3}$ & $5.51765 \times 10^{-7}$ \\
\hline 0.640625 & 0.59769663453 & 0.603940430592 & 0.59769547162 & $6.2438 \times 10^{-3}$ & $1.16292 \times 10^{-6}$ \\
\hline 0.765625 & 0.69298772724 & 0.69859666112 & 0.69298567393 & $5.60893 \times 10^{-3}$ & $2.05332 \times 10^{-6}$ \\
\hline 0.890625 & 0.77746497824 & 0.78235116319 & 0.77746171413 & $4.88618 \times 10^{-3}$ & $3.26411 \times 10^{-6}$ \\
\hline
\end{tabular}
exact, BPF, and IBPF solutions at different points within the interval $[0,1)$. Notice that the modification done to the BPF made the absolute error smaller than the regular BPF. Notice that the collocation points are taken as the midpoints of the subintervals of the IBPF.

Table 5. The numerical results of example 3 at $m=16,32$ and 64 .

\section{Example 4} [18],

$$
y(t)=-\frac{2}{15}-\frac{83}{30} t+\frac{50}{21} t^{2}+\int_{0}^{1}\left(1+s t+s^{2} t^{2}\right)(y(s))^{2} d s
$$

where the exact solution is $y(t)=1-2 t+3 t^{2}$.

Let

$$
y(t) \simeq Y^{T} \Psi_{(m)}(t)
$$




$$
\begin{gathered}
-\frac{2}{15}-\frac{83}{30} t+\frac{50}{21} t^{2} \simeq F^{T} \Psi_{(m)}(t) \\
1+s t+s^{2} t^{2} \simeq \Psi_{(m)}^{T}(s) K_{(m)} \Psi_{(m)}(t) \\
y(t)]^{2} \simeq \tilde{Y}^{T} \phi_{m}(t),
\end{gathered}
$$

where

$$
\tilde{Y}=\left[y_{0}^{2}, y_{1}^{2}, \cdots, y_{m-1}^{2}, y_{m}^{2}\right]
$$

and $Y^{T}=\left[y_{0}, y_{1}, \ldots, y_{m}\right]$ are the undetermined coefficients for the unknown function $y(t), F^{T}=\left[f_{0}, f_{1}, \ldots, f_{m}\right]$ are known and found by using

$$
f_{i}=\left\{\begin{array}{l}
2 m \int_{0}^{1 / 2 m}-\frac{2}{15}-\frac{83}{30} t+\frac{50}{21} t^{2} d t, i=0 \\
m \int_{\frac{i-1 / 2}{m}}^{\frac{i+1 / 2}{m}}-\frac{2}{15}-\frac{83}{30} t+\frac{50}{21} t^{2} d t, 1 \leq i \leq m-1 \\
2 m \int_{1-1 / 2 \mathrm{~m}}^{1}-\frac{2}{15}-\frac{83}{30} t+\frac{50}{21} t^{2} d t, i=m
\end{array}\right.
$$

Also, $K_{(m)}=K_{m+1 \times m+1}$ can be found by using the following relation:

$$
K_{(m)} \simeq\left[k_{i j}\right]_{(m+1 \times m+1)}
$$

Substituting in Eq. (134)

$$
Y^{T} \Psi_{(m)}(t)=F^{T} \Psi_{(m)}(t)+\int_{0}^{t} \Psi_{(m)}^{T}(s) K_{(m)} \Psi_{(m)}(t) \Psi_{(m)}^{T}(t) Y
$$

Then we have the system

$$
Y_{m}=F_{m}+K V \tilde{Y},
$$

By solving this system of linear equations, the improved block pulse series coefficients can be found. After substituting into Eq. (134), the IBPF approximate solution will be found. Now, the following tables show the values of the exact, BPF, and IBPF solutions at different points within the interval

\begin{tabular}{|c|c|c|c|c|c|}
\hline \multirow{2}{*}{$\mathbf{t}$} & \multirow{2}{*}{ Exact solution } & \multirow{2}{*}{ BPF } & \multirow{2}{*}{ IBPF } & \multicolumn{2}{|l|}{ Absolute error } \\
\hline & & & & IBPF & BPF \\
\hline 0.125 & 0.796875 & 0.74395255813 & 0.8083499317 & $5.29224 \times 10^{-2}$ & $1.14749 \times 10^{-2}$ \\
\hline 0.25 & 0.6875 & 0.68110821524 & 0.6987662714 & $6.39178 \times 10^{-3}$ & $1.12663 \times 10^{-2}$ \\
\hline 0.375 & 0.671875 & 0.71184740027 & 0.6828093009 & $3.99724 \times 10^{-2}$ & $1.09343 \times 10^{-2}$ \\
\hline 0.5 & 0.75 & 0.83617011321 & 0.7604790205 & $8.61701 \times 10^{-2}$ & $1.0479 \times 10^{-2}$ \\
\hline 0.625 & 0.921875 & 1.05407635405 & 0.9317754300 & $1.32201 \times 10^{-1}$ & $9.90043 \times 10^{-3}$ \\
\hline 0.75 & 1.1875 & 1.36556612281 & 1.1966985294 & $1.78066 \times 10^{-1}$ & $9.19853 \times 10^{-3}$ \\
\hline 0.875 & 1.546875 & 1.77063941948 & 1.5552483188 & $2.23764 \times 10^{-1}$ & $8.37332 \times 10^{-3}$ \\
\hline
\end{tabular}
$[0,1)$. Notice that the modification done to the BPF made the absolute error smaller than the regular BPF. Notice that the collocation points are taken as the midpoints of the subintervals of the IBPF. This table shows the errors between exact, BPF, and IBPF solutions. It's obvious that the IBPF solution is better than that of BPF.

Table 6. The numerical results of example 8 at $m=8$.

This method is extended to be coupled with other known methods as done in the work by Ramadan and Osheba, [19]. It gave very accurate results that can be used to develop this work thoroughly.

\section{Conclusion}

The IBPFs, also the operational matrices B and $\mathrm{V}$ are used to get numerical solutions of linear and nonlinear Volterra and Fredholm integral equations. The mentioned method reduces the integral equations into an algebraic matrix equation. After solving the matrix equation, we can get the solution easily. The operational matrices have many zeros which make them easier to deal with than other methods. When this method is compared to the original BPF method, it shows a high accuracy at the midpoint of its intervals. This accuracy is much better than that of the original technique. It is stated by the graphs in the numerical applications section. Also, the convergence proved in the current article for the proposed method. The absolute error is shown to state the applicability and accuracy of the method. The article is compared with the work done with many other methods to prove the effectiveness and convenience of the method. It is worth mentioning that the method is extended to solve nonlinear Volterra and Fredholm integral equations.

\section{Aknowlegements}

The authors would like to thank the review and the editor for their accurate revision of this paper and their precious remarks which enhanced the work in this paper.

\section{References}

[1] B. G. Pachpatta, On mixed Volterra-Fredholm type integral equations, Indian Journal of Pure and Applied Mathematics, 17 (4), 488-496 (1986).

[2] E. Yusufoglu, Numerical expansion methods for solving systems of linear integral equations using interpolation and quadrature rules, International Journal of Computer Mathematics, 84 (1), 133-149 (2007).

[3] M. A. Ramadan, G. M. Moatimid, M. H. Taha, A powerful method for obtaining exact solutions of Volterra integral equation's types, Global Journal of Pure and Applied Mathematics, 16 (2), 325-339 (2020). 
[4] K. Maleknejad and F. Mirzaee, Numerical solution of linear Fredholm integral equations system by rationalized Haar function method, International Journal of Computer Mathematics, 80 (11), 1397-1405 (2003).

[5] K. Maleknejad, F. Mirzaee, and S. Abbasbandy, Solving linear integrodifferential equations system by using rationalized Haar function method, Applied Mathematics and Computation, 155 (2), 317-328 (2004).

[6] R. R. Rizkalla, S. Sh. Tantawy, M. H. Taha, Application on differential transform method for some non-linear functions and for solving Volterra integral equations involving fresnel's integral, Journal of Fractional Calculus and Applications, 5 (23), 1-14 (2014).

[7] J. Pour-Mahmoud, M. Y. Rahimi-Ardabili, and S. Shahmorad, Numerical solution of the system of Fredholm integrodifferential equations by the Tau method, Applied Mathematics and Computation, 168 (1), 465-478 (2005).

[8] JH. He, Homotopy perturbation technique. Computer Methods in Applied Mechanics and Engineering, 62, 178-257 (1999).

[9] JH. He, A coupling method of homotopy technique and perturbation technique for nonlinear problems. International Journal of Non-Linear Mechanics, 35 (1), 37-43 (2000).

[10] JH. He, Application of homotopy perturbation method to nonlinear wave equations, Chaos, Solitons and Fractals, 26 (3), 695-700 (2005).

[11] A. M. Wazwaz., Linear and Non-linear Integral equations (methods and applications), Saint Xavier University, Chicago (2011).
[12] F. Mirzaee, Numerical solution of system of linear integral equations via improvement of block-pulse functions, Journal of Mathematical Modeling, 4 (2), 133-159 (2016).

[13] Z. H. Jiang and W. Schaufelberger, Block Pulse functions and their applications in control systems, Spriger-Verlag, Berlin, (1992).

[14] W. Rudin, Principles of Mathematical Analysis, McGraw-Hill, Singapore (1976).

[15] K. Maleknejad, S. Sohrabi and B. Baranji, Application of 2D-BPFs to nonlinear integral equations, Communications in Nonlinear Science and Numerical Simulation, 15 (3), 527-535 (2010).

[16] K. Maleknejad, Y. Mahmoudi, Numerical solution of linear Fredholm integral equation by using hybrid Taylor and block-pulse functions, Applied Mathematics and Computation, 149 (3), 799-806 (2004).

[17] K. Maleknejad, K. Mahdiani, Iterated block-pulse method for solving Volterra integral equations, Applied Mathematics, 2 (1), 17-20 (2012).

[18] A. Shahsavaran, Computational method to solve nonlinear integral equations using block pulse functions by collocation method, AppliedMathematical Sciences, 5 (65), 3211 - 3220 (2011).

[19] Mohamed Ramadan, Heba S. Osheba, A new hybrid orthonormal Bernstein and improved block-pulse functions method for solving mathematical physics and engineering problems, Alexandria Engineering Journal, 5 (59), 3643-3652, (2020). 